\title{
Correction to: Antenna Selection in TDD Massive MIMO Systems
}

\section{Guiyue Jin $^{1} \cdot$ Chaoyue Zhao ${ }^{1} \cdot$ Zhuyun Fan $^{1} \cdot$ Jiyu Jin ${ }^{1}$}

Published online: 18 February 2020

(C) Springer Science+Business Media, LLC, part of Springer Nature 2020

\section{Correction to: Mobile Netw Appl}

https://doi.org/10.1007/s11036-019-01297-5

The original version of this article unfortunately contained mistakes in the "author group", "System model" and "Antenna selection method based on energy efficiency" sections. Author Jiyu Jin's given name was incorrectly spelled as "Jinyu".

The vectors $\mathbf{g}$ and $\mathbf{h}$ are not in bold, the superscript and subscript of element $p$ in vector $\mathbf{P}^{(u l)}=\left[p_{1}^{(u l)}, p_{2}^{(u l)}, \ldots, p_{K}^{(u l)}\right]^{T}$, $p_{i}^{(u l)} \geq 0$ was incorrectly presented as: $\mathbf{P}^{(u l)}=\left[p^{(u l)} 1, p^{(u l)} 2, \ldots\right.$, $\left.p^{(u l)} K\right]^{T}, p^{(u l)} i \geq 0$.

While, the inline equation " $\mathbf{k}_{i}=\underset{l \in\{1,2, \ldots, M / 2\}}{\arg }\left\|\left(\mathbf{W}_{\mathbf{i}}\right)_{l}\right\|^{2}$ " under Step 1 of antenna selection method description was incorrectly presented as: $\mathbf{k}_{i}=\arg _{l \in 1,2, \ldots, M / 2\left\|\left(\mathbf{w}_{i}\right)\right\|_{l} \|^{2}}$.

The original article has been corrected.

The online version of the original article can be found at https://oi.org/ 10.1007/s11036-019-01297-5

Jiyu Jin

jiyu.jin@dlpu.edu.cn

1 School of Information Science and Engineering, Dalian Polytechnic University, Dalian, China 